\title{
A FOLLOW-UP STUDY OF 250 PATIENTS SUBJECTED TO SURGICAL TREATMENT OF PATENT DUCTUS ARTERIOSUS
}

BY

\author{
NILS HELSINGEN, jr., ODD HUSOM, AND LEIF EFSKIND \\ From the Surgical University Clinic A, Rikshospitalet, Oslo, Norway
}

(RECEIVED FOR PUBLICATION APRIL 30, 1958)

Since the first operation for patent ductus arteriosus performed by Gross in 1938, many papers have been published, Gross himself reporting more than 600 patients treated until 1953.

Wide indications for operative treatment are now generally accepted in view of the low operative mortality and the risks that are present without surgery. As to the operation itself, discussion still continues because of the reported tendency for the duct to recanalize after simple ligation.

\section{MATERIAL}

In our department a total of 250 patients suffering from patent ductus arteriosus have been operated upon between 1944 and 1956. Part of the material has been published in a previous report (Liavaag, 1949).

The male-female ratio in this material is exactly the same as that found by Ekström (1952), namely $23 \%$ males. These figures vary somewhat in the different statistics, but the preponderance of females is clear.

The information in our material is scarce concerning the familial incidence, but two sisters have been operated upon, and a woman has a son who has a patent duct. The mother of another patient was later treated for the same defect and the grandmother is also said to have a patent duct, although this has not been verified. Moreover, two of the patients are distant relatives, and in other cases some hints of "heart defect" might indicate additional familial cases. According to Ekström, who reports a verified familial occurrence of $4.5 \%$, only a few writers have described more than one case of patent duct in the same family.

\section{SYMPTOMS}

The frequency of symptoms in patients with patent ductus arteriosus is rather difficult to evaluate for several reasons. As is well known, the symptoms may be very slight and indefinite, such as increased lassitude and irritability. We also repeatedly have had the experience of the mother reporting that her child had "changed completely after operation with an unexpected increase in height and weight," although any symptom had been denied before operation. For orientation we have listed 110 patients as having "no symptoms," 95 patients who complained of "slight symptoms," and 45 with "severe symptoms." Tendency to respiratory infections is well known in association with congenital septal defects, but does not seem to be recognized as a complication of patent ductus arteriosus. In all, 58 of our patients had been treated once or more for "pneumonia." Nineteen patients who belonged to the " no symptom" group had a history of "pneumonia."

Two patients had suffered from subacute bacterial endarteritis before operation, one a boy 8 years old with a combined congenital defect (septal defect and aortic coarctation in addition) who developed sepsis after pneumonia, and the other a woman, 24 years of age, who had the same complication following a spontaneous abortion. Both were treated with penicillin and successfully operated upon. Most of the adult patients coming to operation have symptoms of cardiac embarrassment, which are seldom seen in childhood.

\section{DiAgnosis}

In more than two-thirds of the patients the cardiac lesion was detected by chance during a routine examination at a child welfare centre or at a school.

The special diagnosis of patent ductus arteriosus in nearly $95 \%$ of the patients could be made by auscultation alone, demonstrating the continuous "machinery" murmur in the second or third intercostal space to the left of the sternum. The murmur was atypical in three of the patients. Additional congenital heart defects made the interpretation of the auscultation finding difficult sometimes.

Of the special diagnostic means, heart catheterization was performed in some patients. This is 
now considered necessary only in patients who have atypical murmurs. As a rule the wide duct usually present in these cases can be demonstrated directly by passing the catheter through the communication. Angiocardiography is seldom necessary.

The differential diagnosis has several aspects. As has been pointed out, the typical "machinery" murmur is a distinctive finding. Exactly the same murmur may be heard in cases of "aorticpulmonary" fistula, and in coronary-pulmonary fistula in some cases of high ventricular septal defect with aortic insufficiency. These possibilities must be borne in mind if the patent duct has not been demonstrated directly. In our material two patients referred for operation with a diagnosis of patent ductus arteriosus turned out to have a "ligamentous duct," and the underlying defects were a high ventricular septal defect and an aneurysm of the sinus of Valsalva respectively.

On the other hand, associated cardiac abnormalities may cause diagnostic difficulties. As a rule the diagnosis of a patent ductus arteriosus can be established and the existence of a defect contraindicating operative closure safely ruled out.

In our material at least six patients had an associated atrial septal defect. Six patients are supposed to have a ventricular septal defect, one of these also has aortic coarctation. In at least six patients a pulmonary stenosis was demonstrated. Three patients had aortic stenosis.

\section{TREATMENT}

Wide indications for operative treatment of patients with patent ductus arteriosus are now generally accepted. Contraindication to operation is found in the child who has cyanosis in addition to the auscultatory sounds of a patent duct. With these findings either the patient is in terminal failure resulting from a large ductus arteriosus, or else there is a ductus acting as a compensatory mechanism for a complicated cardiovascular malformation.

What is the "optimum age" for operation? The patent duct seldom ceases spontaneously after 4 or 5 years of age, and as the operation is most safely and easily done in childhood, the patient should be operated upon between 4 and 7 years of age. But the surgeon still has excellent vessels to work upon until 20 years, and in women even later. And in general, if there are definite indications for operation in a small child, such as an enlarged heart with a wide hilum, then therapy should be undertaken with complete dis- regard for the age and size of the patient. Our youngest patient was a boy 14 months old who had an enlarged heart and a wide duct. In the older age groups the symptoms and signs of the patient have to be weighed against the increased surgical risk. Our oldest patient was a woman of 40 years of age.

\section{Surgical Treatment in the Present Series}

Except for the first two patients who were operated upon, all had the usual dorsolateral transthoracic incision with wide opening of the thorax. After the usual dissection the procedure has been simple ligation of the ductus with two or three thick silk ligatures applied as far apart as possible. Sclerosing fluids have not been used. In five cases the duct was cut between clamps and sutured, and in four others sutures had to be placed on the aortic and pulmonary ends of the duct, but without division. That is true also in the case of the 40-year-old woman who showed collar-formed calcification of the duct at its origin from the aorta, and here a division of the duct with sutures would have been difficult.

\section{Surgical Complications}

The mortality in this series was $1.2 \%$. Of the three fatal cases one was attributable to accidental bleeding during the primary operation, the other occurred during re-operation for a complicating aneurysm, and the third death was due to a tension pneumothorax developing after a simple thoracocentesis.

Large but not fatal arterial haemorrhage occurred in four patients because the ligatures cut through.

Pleural effusion and pneumothorax have been constant findings after operation, but as a rule without importance. Thoracocentesis in our material has been performed in 34 patients. One of them was a girl who died from tension pneumothorax.

Atelectasis of clinical importance has been detected in 10 patients (with regular radiological control of all patients).

"Pneumonia" has been found in six patients in the post-operative period, a moderate number considering the reported tendency to respiratory infections before operation.

Pericarditis has been found in one patient who was operated upon through the anterior incision and who sustained a lesion of the pericardium during operation.

Subacute bacterial endarteritis was diagnosed in two patients in the post-operative period. 
No case of post-operative arterial embolism or thrombosis was seen.

Paralysis of the left recurrent nerve has been a complication in eight patients. Although this paralysis is known to occur as a spontaneous complication, it was never diagnosed before operation in our patients, and it must be listed as a surgical lesion in these eight patients, i.e., about $3 \%$. In four of these the operation was described as uneventful ; in the other four adhesions or accidental bleeding made dissection of the duct difficult.

\section{Late Results}

The material for the follow-up examination comprised 250 patients with an observation time varying between six months and 13 years. All the patients were traced; in four cases a control examination could not be obtained. These four patients are reported to be living and in good health.

As is known from the statistics of large series, the results following operative treatment of patent ductus arteriosus are extremely satisfactory at least as far as the patients themselves and their relatives have been able to judge. This is also our impression from this follow-up study. And we repeatedly have had the experience of the mother telling us that her child had changed completely after operation, although symptoms had been denied pre-operatively.

The crucial point in the follow-up examination is the detection of patients with possible recanalization of the ductus arteriosus. In four cases a continuous murmur of the "patent duct type " could be heard at the follow-up examination indicating recanalization. Case reports follow:

CASE 1.-A 29-year-old pregnant woman was operated upon in 1947. She had a medium-sized patent duct, and two silk ligatures were placed. No complication was described, and no murmur could be heard at the post-operative examination. One year later a loud systolic murmur was registered, and at the follow-up a typical continuous murmur was heard. She has slight symptoms of cardiac embarrassment.

CASE 2.-A girl aged 8 years had a patent ductus arteriosus (diameter $3 \frac{1}{2} \mathrm{~cm}$., length $\frac{1}{2} \mathrm{~cm}$.) when operated upon in 1952. A few days after operation the continuous murmur recurred although it was weaker than before. Her post-operative recovery was delayed by persistent fever and haematuria, most probably indicating a subacute bacterial endarteritis. In 1953 a re-thoracotomy was performed showing a thin-walled pulsating aneurysm, the size of a mandarin orange, at the site of the previous ductus arteriosus and probably communicating with the pulmonary artery and aorta. At the follow-up examination she was found to have moderate symptoms of cardiac embarrassment (blood pressure in the right arm $125 / 30 \mathrm{~mm}$. Hg). Radiography showed calcification of the aneurysm.

CASE 3.-The third patient was a boy 12 years old when operated upon in 1953 for a wide ductus arteriosus (diameter about $4 \mathrm{~cm}$.). A continuous murmur could be heard one week post-operatively although it was weaker than before operation. The findings are now the same. $\mathrm{He}$ is moderately handicapped.

CASE 4.-The fourth patient was a woman of 23 years of age when operated upon in 1953 for a wide ductus arteriosus. Probably she already had a continuous murmur at post-operative control. At the follow-up a typical " machinery" murmur was heard, and heart catheterization performed indicated a " $25 \%$ shunt."

Thus four patients showed signs of recanalization following simple ligation of the ductus arteriosus. Two of them were more than 20 years of age at operation. Three had wide ducts, and one probably had a subacute bacterial endarteritis post-operatively.

The incidence of recurrence in the present material is $1.6 \%$. This compares favourably with the reports of some other authors of more than $10 \%$ recanalization after ductus ligation.

Under these conditions we regard ligation as a safe method with a small chance of recurrence, except in cases of giant ducts and in elderly patients.

\section{SUMMARY}

The study presented is based on 250 patients with patent ductus arteriosus, the greater part treated with simple ligation.

The general aspects of the cases studied are given and a discussion of some cardinal points. A high incidence of pneumonia is reported before operation. Three operative or post-operative deaths occurred, i.e., a surgical mortality of $1.2 \%$.

A report is given of the late results, especially of the incidence of recanalization of the ductus arteriosus following operation. Four patients showed recurrence, i.e., a frequency of $1.6 \%$.

Under these conditions simple ligation is considered a safe procedure with a small chance of recurrence, except in cases of giant ducts and in elderly patients.

\section{REFERENCES}

Ekström, G. (1952). Acta chir. scand., Suppl. 169.

Gross, R. E. (1953). The Surgery of Infancy and Childhood. W. B.

Saunders, Philadelphia.
Liavaag, K. (1949). Acta chir. scand., 98, 109. 